\title{
Sonographic Bedside Quantification of Pleural Effusion Compared to Computed Tomography Volumetry in ICU Patients
}

\section{(두)(i) (ㅇ) $\ominus$}

Authors

Ulf Karl-Martin Teichgräber ${ }^{1,3}$, Judith Hackbarth²,3

\section{Affiliations}

1 Institut für Diagnostische und Interventionelle Radiologie, Universtätsklinikum Jena, Jena, Germany

2 Thoraxklinik, Abteilung für Anästhesiologie und Intensivmedizin, Universitätsklinikum Heidelberg, Heidelberg

3 Institut für Diagnostische und Interventionelle Radiologie, Charité Universitätsmedizin Berlin, Berlin, Germany

\section{Key words}

pleural effusion, sonography, volumetric assessment, chest $C T$

$\begin{array}{ll}\text { received } & 21.12 .2017 \\ \text { revised } & 27.07 .2018 \\ \text { accepted } & 30.08 .2018\end{array}$

\section{Bibliography}

DOI https://doi.org/10.1055/a-0747-6416

Ultrasound Int Open 2018; 4: E131-E135

(c) Georg Thieme Verlag KG Stuttgart · New York

ISSN 2199-7152

Correspondence

Prof. Ulf Karl-Martin Teichgräber, MD, MBA

Institut für Diagnostische und Interventionelle Radiologie

Universtätsklinikum Jena

Am Klinikum 1

07747 Jena

Germany

Tel.: +49/03641/9324 831, Fax: +49/03641/9324832

ulf.teichgraeber@med.uni-jena.de

\section{ABSTRACT}

Objectives To date, the reliability of ultrasound for the quantitative assessment of pleural effusion has been limited. In the following study, an easy and cost-effective bedside ultrasound method was developed and investigated for specific use in the intensive care unit (ICU).

Methods 22 patients (median age: 58.5 years, range: $37-88$ years, 14 men and 8 women) with a total of 31 pleural effusions were examined in the ICU. The inclusion criterion was complete visualization of the effusion on chest computed tomography (CT). The ultrasound (US) examination was performed less than $6 \mathrm{~h}$ after the diagnostic CT scan. The pleural effusion volume was calculated volumetrically from the CT scan data. Within $4.58+/-2.87 \mathrm{~h}$ after the CT scan, all patients were re-examined with US in the ICU. The fluid crescent's thickness was measured between each intercostal space (ICS) with the patient in a supine position and $30^{\circ}$ inclination of the torso. The US measurements were compared to the calculated CT volumes using regression analysis, resulting in the following formula: $V=13.330 \times \operatorname{ICS} 6(\mathrm{~V}=$ volume of the effusion [ $\mathrm{ml}]$; ICS6 = sonographic measurement of the thickness of the liquid crescent [mm] in the sixth ICS).

Results A significant correlation between the sonographically measured and the CT-calculated volumes was best observed for the sixth ICS $(R 2=0.589$; ICC $=0.7469$ with $p<0.0001$ and a $95 \% \mathrm{Cl}$ of $0.5364-0.8705)$.

Conclusion The sonographic assessment of pleural effusions in a supine position and $30^{\circ}$ inclination of the torso is feasible for the volumetric estimation of pleural effusion. This is especially true for ICU patients with severe primary diseases and orthopnea who are unable to sit upright or lie flat.

\section{Introduction}

Chest sonography is a viable bedside method to verify free fluid and to differentiate contained effusions, pleural peel, atelectasis, diaphragmatic elevation, and other lesions [1-5]. To date, there is no reliable method for the fast quantification of pleural effusions in intensive care unit (ICU) patients as a diagnostic basis for puncture or drainage.
In 1994, Eibenberger et al. developed a sonographic method for the volumetric quantification of pleural effusions in a strictly supine position [6]. In this study, the extent of the effusion was measured sonographically in each intercostal space. From these measurements and the effusion volumes, which were quantified by puncture, a formula was developed to estimate effusion volumes. 
Such methods are feasible in ICU patients because they are rarely able to sit upright [6, 7]. This is especially true for consciously sedated and ventilated patients. Patients in respiratory distress who are not intubated and often ventilated suffer from orthopnea and are unable to lie flat on their back. Schmidt et al. developed a method for the estimation of pleural effusion volumes in patients with a $30^{\circ}$ inclination of the torso [8]. The pleural effusion volume was defined as the sum of the basal expanse of the free fluid between the diaphragm, the inferior lobe of the lung, and the maximum craniocaudal expanse of the effusion multiplied by a factor of 70 . This method is adequate and feasible for ICU patients with a slight inclination of the torso. However, this approach requires multiple measurements and is therefore relatively time-consuming and less feasible in an ICU setting.

The aim of this prospective non-randomized study was to develop a simple and fast method for the bedside sonographic quantification of pleural effusions in ICU patients.

\section{Materials and Methods}

\section{Patient cohort}

Overall, 22 patients (median age: 58.5 years, range: $37-88$ years, 14 male and 8 female patients) with a total of 31 pleural effusions were sonographically examined in a university hospital. A chest CT was performed on all patients as part of the clinical and diagnostic routine prior to the ultrasound exam. All CT scans were then assessed and evaluated by an experienced radiologist. If pleural effusions were detected, the patient was re-examined with an additional sonogram. The inclusion criteria demanded that the chest $\mathrm{CT}$ and the ultrasound exam be performed less than six hours apart and that the patient had not yet been treated with effusion puncture or drainage prior to the ultrasound exam. Finally, only patients with uncontained effusions were included in this study.

\section{Sonographic measurements of pleural effusion}

The sonographic exam was performed while the patient's torso was positioned at a $30^{\circ}$ incline. Intercostal spaces (ICSs) 4-9 were traced in the posterior axillary line (PAL) with a 3.5-MHz curved linear transducer head of an ultrasound unit type EUB 405 (Hitachi Medical Systems, Tokyo, Japan). The ICSs were identified on the basis of anatomical landmarks such as the claviculae and vertebral bodies and used as acoustic windows. The transducer head was tilted until the effusion became visible on the ultrasound screen. The expanse of the fluid moat between the visceral pleura and the parietal pleura was measured in millimeters. The most caudal portion of the effusion was chosen for each measurement ( Fig. 1).

\section{Computed tomographic volumetry}

The patients were selected on the basis of routine diagnostic CT scans, which depicted the entire pleural effusion. All scans were performed on a 4-slice spiral CT scanner with a slice thickness of $5 \mathrm{~mm}$ and a pitch of 2 . The pleural effusion volume was calculated using semi-automated volumetric software with threshold analysis and contour limiting on an Easyvison workstation (Philips Healthcare, Best, the Netherlands). A threshold analysis was used to subtract the air and skeletal structures from the calculated volume. Each image slice was examined and evaluated individually. The area of effusion was delineated with the cursor in each image slice by the radiologist ( $>$ Fig. 2 ). The computer program then calculated the total volume of the pleural effusion from the sum of each demarcated region and slice thickness.

\section{Statistical analysis}

The sonographically measured values in millimeters were compared to the CT-calculated volumes in a scatter plot. The CT volumes and the maximum extent of the effusion were compared using linear regression analysis. The regression line equations were then used to calculate the pleural effusion volumes, which were then compared to the corresponding CT-calculated volumes. An interclass correlation coefficient (ICC) was determined for each comparison. On the basis of the regression line function of the ICS

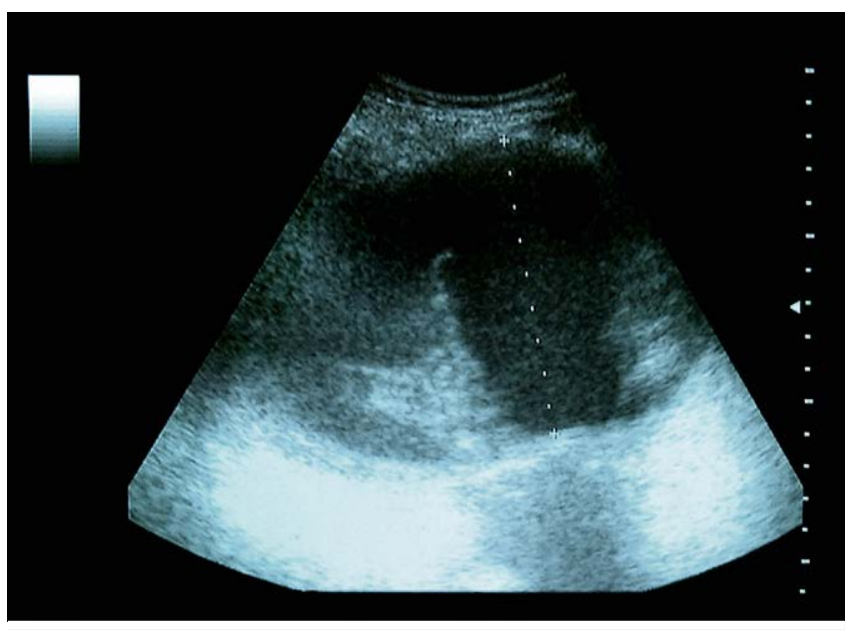

-Fig. 1 Measurement of the liquid crescent of the pleural effusion during sonography.

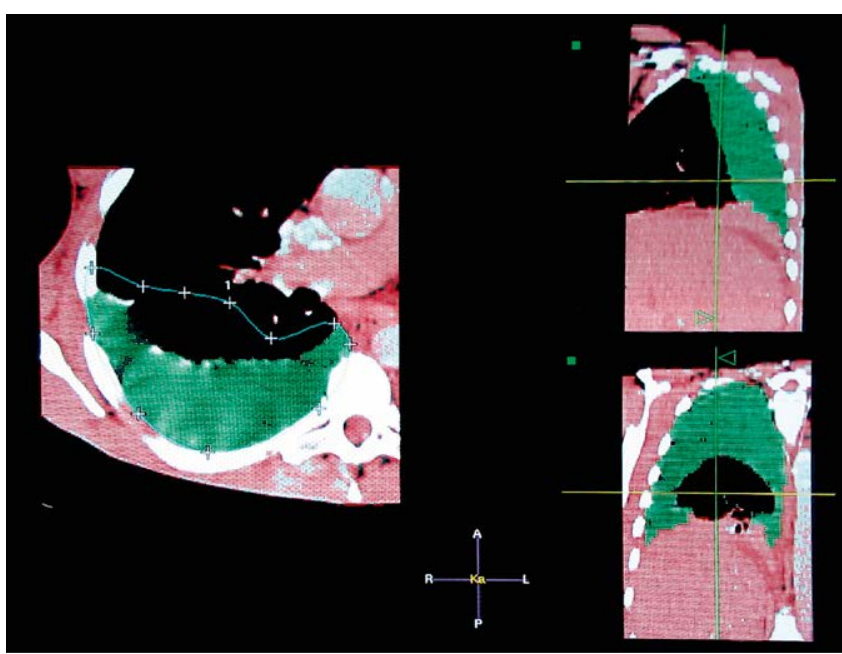

- Fig. 2 CT reconstruction in 3 planes: Region marked with cursor (green: volume considered in total volume calculation) in a single CT image slice after threshold analysis (red: volume not considered for total volume calculation). 
with the highest ICC and the smallest two-way confidence interval $(\mathrm{Cl})$, a formula was developed to calculate the pleural effusion volumes with the sonographic image data.

\section{Results}

To assess and measure the maximum interpleural expanse of the effusions, a sonographic window at the sixth ICS in the PAL is ideal. Both the $\mathrm{Cl}$ and ICC for the sixth ICS as sonographic windows confirm this hypothesis in > Fig. 3.

The sixth ICS regression line equation is as follows:

$$
\text { Volume }_{\mathrm{CT}}(\mathrm{ml})=13.330 \times \operatorname{section}_{\text {ultrasound }}(\mathrm{mm})+27.134
$$

The regression coefficient for the regression line of the sixth ICS was $r=0.767$. The coefficient of determination was $R^{2}=0.589$ with a $95 \% \mathrm{Cl}$ of $9.017-17.643$ of the regression line. The ICC for the sixth ICS was 0.7487 ( $\mathrm{p}<0.00001 ; 95 \% \mathrm{Cl} 0.5393-0.8714$ ).

Because the absolute coefficient loses its mathematical relevance in practice, the absolute coefficient is negligible in favor of a simplified formula:

$$
\mathrm{V}_{\text {effusion in CT }}(\mathrm{ml})=13.330 \times \text { thickness }_{\text {liquid head in the sixth ICS }}(\mathrm{mm})
$$

We have listed the rounded values with which the estimation of the pleural effusion volume is more comfortable and considered the standard deviation in $>$ Table 1.

\section{Discussion}

In the verification of pleural effusions, sonography (sensitivity: $100 \%$, specificity: $99.7 \%$ ) is superior to chest X-ray in an erect patient (sensitivity: $71 \%$, specificity: $98.5 \%$ ) [1, 9]. Moreover, an upright chest X-ray is impossible for most ICU patients with severe primary diseases and impaired physical stamina. The sonographic identification of pleural effusions is simple; the effusions appear

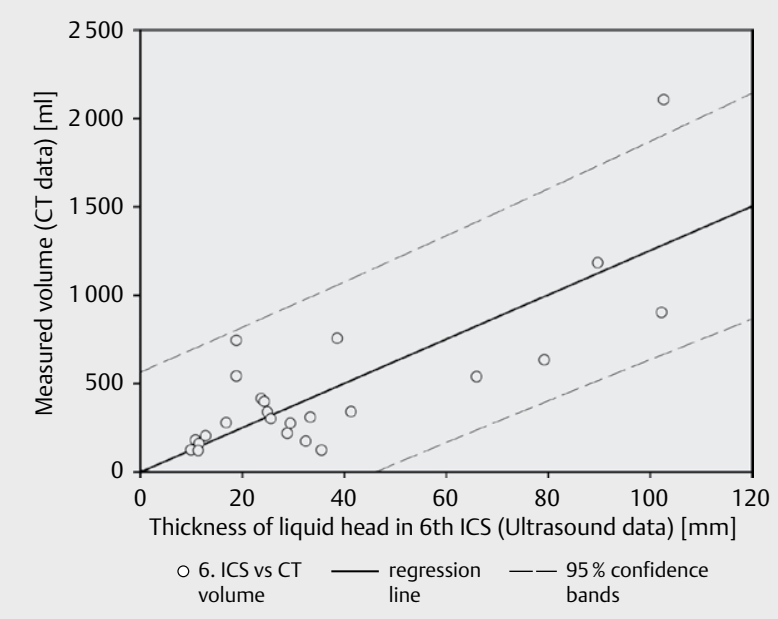

- Fig. 3 Regression diagram for the 6th ICS: measured volume (CT data) over thickness of liquid head in the 6th ICS (ultrasound data). echo-free, are sharply delineated, and show dorsal echo enhancement [2,3]. Since atelectasis, diaphragmatic elevation, tumors, and pleural sheaths cause no difficulty with respect to differentiation on ultrasound images as in conventional fluoroscopic images, larger effusions can be diagnosed effortlessly [1, 2, 5]. An effusion volume of at least $150 \mathrm{ml}$ is necessary for the diagnosis of an uncontained pleural effusion on a standard $X$-ray in an erect patient [10]. However, 5-ml effusion volumes in the basal and laterodorsal compartment between the ribcage and the diaphragm suffice for a reliable sonographic diagnosis [10]. The ultrasound examination can be performed bedside and can be repeated easily and quickly for control purposes. Compared to conventional chest X-ray, sonography is the more precise method and is also readily available in most ICUs [6].

In this study, a sonogram was performed on patients whose torso was inclined by $30^{\circ}$. The image data were collected during expiration. In 1994, Eibenberger et al. examined 51 patients with pleural effusions in a strictly supine position at maximum inspiration. In this study, the expanse of the pleural effusion was measured sonographically. Measurements were taken between the parietal and visceral pleura. In the second step, the effusion was punctured in 200-ml increments until the entire effusion was drained. Sonographic measurements were taken between each puncture from the same angle. All 331 sonographic measurements were then compared to the drained volumes using regression analysis. Statistical analysis rendered the following formula: $y=47.6 \times-837$ (the formula proposed in this paper is $V=13.330 \times$ ICR6) with $y$ equaling the approximated effusion volume $(\mathrm{ml})$ and $\mathrm{x}$ being the maximum expanse of the effusion $(\mathrm{mm})$. The arithmetic median standard deviation was $+1-224 \mathrm{ml}[6]$.

In a further study, Balik et al. examined 81 patients with pleural effusions in a supine position with a torso elevation of $15^{\circ}$. The maximum separation between the parietal and visceral pleura (Sep) at maximum expiration was measured. Thoracocentesis was performed

- Table 1 Estimation of effusion volume with sonographically measured liquid crescent.

\begin{tabular}{|l|c|c|}
\hline $\begin{array}{l}\text { Thickness of liquid } \\
\text { crescent in } \\
\text { sonography } \mathbf{( m m )}\end{array}$ & $\begin{array}{c}\text { Estimated volume } \\
\text { of the effusion } \\
\mathbf{( m \mathbf { l } )}\end{array}$ & $\begin{array}{c}\text { Standard } \\
\text { deviation } \mathbf{( m )}\end{array}$ \\
\hline 5 & 70 & 20 \\
\hline 10 & 130 & 40 \\
\hline 15 & 200 & 60 \\
\hline 20 & 270 & 90 \\
\hline 30 & 400 & 130 \\
\hline 40 & 530 & 170 \\
\hline 50 & 670 & 220 \\
\hline 60 & 800 & 260 \\
\hline 70 & 930 & 300 \\
\hline 80 & 1070 & 350 \\
\hline 90 & 1200 & 390 \\
\hline 100 & 1330 & 430 \\
\hline
\end{tabular}


and the total volume of the drained effusion was determined. The pleural effusion was calculated as follows: $\mathrm{V}(\mathrm{ml})=20$ * Sep $(\mathrm{mm})$. The mean predictive error of $V$ in this method was $158 \mathrm{ml} \pm 160.6 \mathrm{ml}$ [11]. For comparison, in our study, the arithmetic median standard deviation was slightly worse at $+/-204.85 \mathrm{ml}$.

However, these values should be seen in a clinical context. An exact quantification, i.e., $260 \mathrm{ml}$ exactly, is most likely less important than the ability to determine if an effusion volume is in the range of $200 \mathrm{ml}$ or $300 \mathrm{ml}$.

Ventilated lateral lung compartments may mask small effusions and thus impede reliable measurement of the effusion, especially during deep inspiration [12]. Therefore, sonographic measurements were performed during expiration. The patients were asked to breathe calmly and normally, with the goal of achieving comparability between spontaneous breathing and ventilated patients.

In this study, CT volumetry was used as a reference volume (the gold standard). We based this decision on studies that showed good correlation between the actual organ volumes and CT-calculated volumes [13-17]. It needs to be further evaluated whether CT calculations render more precise results than drainage in the quantification of effusion volumes. After the puncture of a pleural effusion, a residual volume may remain within the interpleural space and could thus lead to an underestimation of the total effusion volume. With CT volumetry, the entire effusion volume is imaged. However, this method can lead to other sources of error, such as the breath-induced movement of the thoracic organs. Since all CT scans were performed in a spiral CT, the patients were asked to hold their breath for 20 to $40 \mathrm{~s}$. The scan technique errors caused by breath-induced movements are negligible [18]. Moreover, outlining the effusion with the cursor in each image slice can induce user-related errors [18]. Through the predefinition of a density threshold in CT excluding air and bone from the soft tissue image, merely intercostal musculature and atelectasis had to be differentiated from the effusion. The intercostal musculature was easy to differentiate due to its anatomic location; atelectases were precluded from the volume due to differences in density on the CT images. Differentiation from the diaphragm and liver in the abdominal slices of the CT images was also achieved using the anatomic location and density.

Drainage of pleural effusions for the diagnostic determination of effusion volume is an invasive method that leads to risks such as bleeding, infection, and pneumothorax. The formula we describe herein for the estimation of pleural effusion volumes with sonography is a dimension-free function describing a regression line. This allows the estimation of an effusion volume from a sonographically measured distance, i. e., the maximum expanse of the effusion measured from the sixth ICS. Further validation of the formula with a larger patient cohort and differentiation between ventilated and spontaneously breathing patients are necessary. To ensure simplicity and clinical feasibility, we assembled rounded measurements of the maximum expanse of the effusion to simplify the clinical quantification ( $\triangleright$ Table $\mathbf{1}$ ).

This technique can be used for bedside examinations in most clinical settings due to the wide availability and mobility of modern ultrasound units $[19,20]$. Sonography of pleural effusions is a feasible method for critically ill patients who are mechanically ventilated [11, 21, 22]. Moreover, a sonographic examination does not expose the patient or physician to ionizing radiation [19]. The ultrasound volumetric estimation of pleural effusions permits both determination of an indication for drainage and determination of the ideal puncture point $[6,8,23,24]$. Furthermore, sonography is a cost-efficient method [19].

Our results are comparable to those noted in the cited studies, which used drainage as the gold standard for quantification. In contrast to the existing literature, we took advantage of the accuracy of CT volumetry and avoided the disadvantages and inaccuracies of drainage.

In conclusion, the ultrasound volumetric estimation of pleural effusion volumes is easy, cost-efficient, and clinically feasible using the proposed method. This method is ideal in critically ill ICU patients for bedside determination of an indication for pleural effusion diagnostics and drainage.

\section{Conflict of Interest}

The authors declare no conflict of interest.

\section{References}

[1] Mathis G. Thoraxsonography-Part I: Chest wall and pleura. Ultrasound Med Biol 1997; 23: 1131-1139

[2] Pernice H, Braun B. Sonographische Differenzierung pulmonaler Verschattungen. Prax Klin Pneumol 1979; 33: 1132-1137

[3] Matalon TA, Neiman HL, Mintzer RA. Noncardiac chest sonography. The state of the art. Chest 1983; 83: 675-678

[4] Rosenberg ER. Ultrasound in the assessment of pleural densities. Chest 1983; 84: 283-285

[5] Doust BD, Baum JK, Maklad NF et al. Ultrasonic evaluation of pleural opacities. Radiology 1975; 114: 135-140

[6] Eibenberger KL, Dock WI, Ammann ME et al. Quantification of pleural effusions: sonography versus radiography. Radiology 1994; 191: 681-884

[7] Lorenz ], Börner N, Nikolaus HP. [Sonographic volumetry of pleural effusions]. Ultraschall Med 1988; 9: 212-215

[8] Schmidt O, Simon S, Schmitt R et al. Volumetrie von Pleuraergüssen bei multimorbiden, postoperativen Patienten einer operativen Intensivstation, Vergleich von Sonographie und Thoraxbettaufnahme. Zentralbl Chir 2000; 125: 375-379

[9] Schwerk WB, Riester KP, Hess F. Real-Time- Ultraschalltomographie von Pleuraergüssen und pleuranahen intrathorakalen Raumforderungen. Respiration 1980; 39: 219-228

[10] Gryminski J, Krakowka P, Lypacewicz G. The diagnosis of pleural effusion by ultrasonic and radiologic techniques. Chest 1976; 70 : 33-37

[11] Balik M, Plasil P, Waldauf P et al. Ultrasound estimation of volume of pleural fluid in mechanically ventilated patients. Intensive Care Med 2006; 32: 318-321

[12] Börner N, Kelbel C, Lorenz J, Weilemann LS, Meyer J. Sonographische Volumenbestimmung und Drainage von Pleuraergüssen. Ultraschall in Klinik und Praxis 1987; 2: 148-152

[13] Moss AA, Friedman MA, Brito AC. Determination of liver, kidney, and spleen volumes by computed tomography: an experimental study in dogs. J Comput Assist Tomogr 1981; 5: 12-14 
[14] Heymsfield SB, Fulenwider T, Nordlinger B et al. Accurate measurement of liver, kidney, and spleen volume and mass by computerized axial tomography. Ann Intern Med 1979; 90: 185-187

[15] Fritschy P, Robotti G, Schneekloth G et al. Measurement of liver volume by ultrasound and computed tomography. J Clin Ultrasound 1983; 11: 299-303

[16] Breiman RS, Beck JW, Korobkin M et al. Volume determinations using computed tomography. AJR Am J Roentgenol 1982; 138: 329-333

[17] Brenner DE, Whitley NO, Houk TL et al. Volume determinations in computed tomography. Jama 1982; 247: 1299-1302

[18] Lemke AJ, Hosten N, Neumann K et al. CT-Volumetrie der Leber vor der Transplantation. Röfo Fortschr Geb Röntgenstr Neuen Bildgeb Verfahr 1997; 166: 18-23

[19] Patel MC, Flower CD. Radiology in the management of pleural disease. Eur Radiol 1997; 7: 1454-1462
[20] Hirsch JH, Rogers JV, Mack LA. Real-time sonography of pleural opacities. AJR Am J Roentgenol 1981; 136: 297-303

[21] Vignon P, Chastagner C, Berkane V et al. Quantitative assessment of pleural effusion in critically ill patients by means of ultrasonography. Crit Care Med 2005; 33: 1757-1763

[22] Roch A, Bojan M, Michelet $P$ et al. Usefulness of ultrasonography in predicting pleural effusions $>500 \mathrm{~mL}$ in patients receiving mechanical ventilation. Chest 2005; 127: 224-232

[23] Opacic M, Bilic A, Ljubicic $\mathrm{N}$ et al. Thoracocentesis under ultrasonographic control. Acta Med lugosl 1991; 45: 71-75

[24] Kohan JM, Poe RH, Israel RH et al. Value of chest ultrasonography versus decubitus roentgenography for thoracentesis. Am Rev Respir Dis 1986; 133: $1124-1126$ 